\title{
Minimum Lens Size Supporting the Leaky-Wave Nature of Slit Dipole Antenna at Terahertz Frequency
}

\author{
Niamat Hussain, ${ }^{1}$ Truong Khang Nguyen, ${ }^{2}$ Haewook Han, ${ }^{3}$ and Ikmo Park ${ }^{1}$ \\ ${ }^{1}$ Department of Electrical and Computer Engineering, Ajou University, Suwon, Republic of Korea \\ ${ }^{2}$ Division of Computational Physics, Institute for Computational Science and Faculty of Electrical-Electronics Engineering, \\ Ton Duc Thang University, Ho Chi Minh City, Vietnam \\ ${ }^{3}$ Department of Electrical and Computer Engineering, POSTECH, Pohang, Republic of Korea
}

Correspondence should be addressed to Ikmo Park; ipark@ajou.ac.kr

Received 9 May 2016; Revised 19 August 2016; Accepted 14 September 2016

Academic Editor: Shih Yuan Chen

Copyright (C) 2016 Niamat Hussain et al. This is an open access article distributed under the Creative Commons Attribution License, which permits unrestricted use, distribution, and reproduction in any medium, provided the original work is properly cited.

We designed a slit dipole antenna backed by an extended hemispherical silicon lens and investigated the minimum lens size in which the slit dipole antenna works as a leaky-wave antenna. The slit dipole antenna consists of a planar feeding structure, which is a center-fed and open-ended slot line. A slit dipole antenna backed by an extended hemispherical silicon lens is investigated over a frequency range from 0.2 to $0.4 \mathrm{THz}$ with the center frequency at $0.3 \mathrm{THz}$. The numerical results show that the antenna gain responses exhibited an increased level of sensitivity to the lens size and increased linearly with increasing lens radius. The lens with the radius of $1.2 \lambda_{o}$ is found to be the best possible minimum lens size for a slit dipole antenna on an extended hemispherical silicon lens.

\section{Introduction}

The congestion of the electromagnetic spectrum at microwave frequencies and the rapidly increasing demand for more bandwidth have attracted researchers to an unexplored spectrum of the terahertz $(\mathrm{THz})$ band. A higher $\mathrm{THz}$ bandwidth has the potential to achieve an extremely high data rate, such as one terabit-per-second, for future wireless devices [1]. In comparison with the conventional cellular systems operating below $2 \mathrm{GHz}$, we can expect that $\mathrm{THz}$ waves in the range of 0.1 to $1 \mathrm{THz}$ can provide bandwidth 50 to 500 times larger [2]. This range is popular for terahertz communications due to the existence of many low-attenuation windows, such as the $300,350,410,670$, and $850 \mathrm{GHz}$ bands. The terahertz frequency range has become very attractive in many other applications, such as radio astronomy, atmospheric research, chemical spectroscopy, medical imaging, security screening, and defense [3-5]. The effective utilization of $\mathrm{THz}$ bands is due to the availability of stable, compact, low cost, and high sensitive sources and detectors. Extensive research has been carried out on lens-coupled antennas (a combination of planer antenna with a quasi-optical lens) for millimeter and submillimeter wave systems to increase the gain by reducing the coupling loss due to the excitation of surface wave modes. The other advantages of this technique include mechanical rigidity, thermal stability, compatibility with IC techniques, and capability for multiple-beam formation in a simpler way.

In the past, lens-coupled antennas, including dipole antennas $[6,7]$, bow-tie antennas [8], annular-slot antennas [9], log-periodic antennas [10], and folded-dipole antennas [11], have been studied. In particular, many researchers focused on the coupling of leaky-wave antennas with lenses due to their important and attractive properties, for instance, their broad impedance bandwidth, high directivity, and simple feeding network [12]. Various leaky dielectric lens antennas fed by a stripline dipole [13], typical leaky-wave slot [14], leaky-wave waveguide [15], and an array of leaky-wave slots [16] have been studied thoroughly in the literature. However, there is no detailed study of a lens with different sizes for slit dipole antenna to determine the optimal minimum lens size for miniaturization.

Owing to the important and attractive properties of leakywave antennas, researchers have coupled these antennas with lenses to combine the characteristics of lens antennas and 


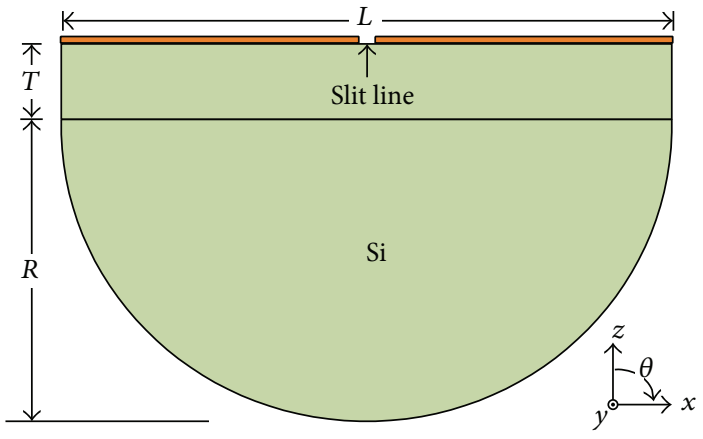

(a)

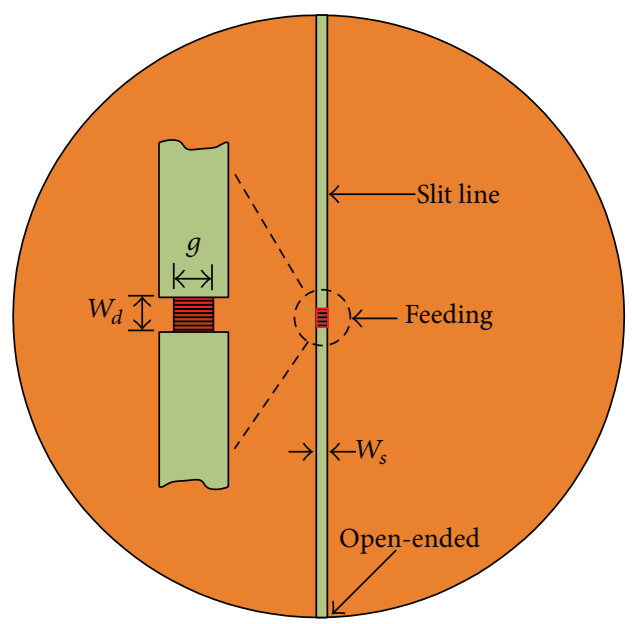

(b)

Figure 1: Antenna geometry: (a) side view and (b) top view.

leaky-wave antennas. In this paper, we designed a slit dipole antenna backed by an extended hemispherical silicon lens and investigated the influence of lens size and shape on the overall characteristics of the antenna at a frequency of around $0.3 \mathrm{THz}$. Based on this investigation, we found the minimum lens size in which an antenna works as a leaky-wave antenna. The investigation was carried out using the finite-integration time-domain simulator from CST Microwave Studio [17], which allows the complete characterization of the performance of lens-coupled antennas at many frequency points with one simulation run. The paper is organized as follows. First, the antenna geometry is described. Second, the antenna characteristics are investigated in detail for different lens sizes and $T / R$ ratios, and finally, conclusions are given.

\section{Antenna Geometry}

Figure 1 shows the detailed geometry of the slit dipole antenna backed by an extended hemispherical silicon lens $\left(\varepsilon_{r}=11.9\right.$ and $\tan \delta=0.0$ ). The slit dipole antenna consists of a leakywave open-ended narrow slit [18] of width $W_{s}$ and length $L$, which is fed by a short dipole at the center. The short dipole has a width and gap of $W_{d}$ and $g$, respectively.

The silicon lens has an extension length and radius of $T$ and $R$, respectively. The antenna is fed by a discrete port having $50 \Omega$ characteristic impedance, placed at the center feed gap. The thickness of metal layer is $0.35 \mu \mathrm{m}$, and its conductivity is $1.6 \times 10^{7} \mathrm{~S} / \mathrm{m}$. The fixed and the dependent parameters used in the study are summarized in Table 1.

The lenses with different radii $(R)$ were selected in the study to determine the minimal lens size, and the shape of the lens determined by $T / R$ ratio (the ratio between the extension length and radius of the lens) was optimized for each lens size by varying the $T / R$ ratio. The lens shape optimization is very important because it plays a key role in maximizing antenna gain and radiation spectral bandwidth in lens-coupled antennas $[19,20]$. Therefore, the optimization of a silicon lens is important and requires careful tuning to
TABLE 1: Design parameters of the antenna.

\begin{tabular}{lc}
\hline Parameter & Dimension $(\mu \mathrm{m})$ \\
\hline$R$ & $L / 2$ \\
$g$ & 10 \\
$W_{s}$ & 20 \\
$W_{d}$ & 10 \\
$T$ & $0.34 R$ \\
\hline
\end{tabular}

achieve the best possible performance. For a fair comparison, the fixed $T / R$ ratio provided the same percentage increase in overall volume of the antenna.

\section{Antenna Characteristics}

The lenses with the radii $(R)$ of $0.8 \lambda_{o}(0.8 \mathrm{~mm}), 1.2 \lambda_{o}$ $(1.2 \mathrm{~mm})$, and $1.6 \lambda_{o}(1.6 \mathrm{~mm})$ were selected for study; $\lambda_{o}$ is the wavelength of the central frequency of $0.3 \mathrm{THz}$ in free space. The shape of each lens determined by the $T / R$ ratio was optimized to obtain a superhemispherical lens by changing the values of $T / R$. For each lens radius, the $T / R$ ratio, which determined the optimized lens shape, was obtained by varying the extension length $(T)$ behind the hemispherical position. Figure 2 shows the antenna gain responses with variations in the $T / R$ ratio in a frequency range from 0.2 to $0.4 \mathrm{THz}$. The $T / R$ ratio was varied from 0.26 to 0.42 in increments of 0.08 . For a lens radius of $0.8 \lambda_{o}$ [Figure 2(a)] the gain curves were similar for wide values of $T / R$; however, a significant difference in gain curves was observed for the other lenses. The gain level for lens radius of $1.2 \lambda_{o}$ [Figure $2(b)$ ] increased when the $T / R$ ratio increased from 0.26 to 0.34 . However, when it further increased to 0.42 , the gain level decreased. The gain curve for $T / R=0.42$ was similar to the gain curve observed at $T / R=0.26$. The similar trend is seen with greater differences in gain curves for the lens radius of $1.6 \lambda_{o}$. This behavior is attributed to the effect of extension length $(T)$ which changes the foci 


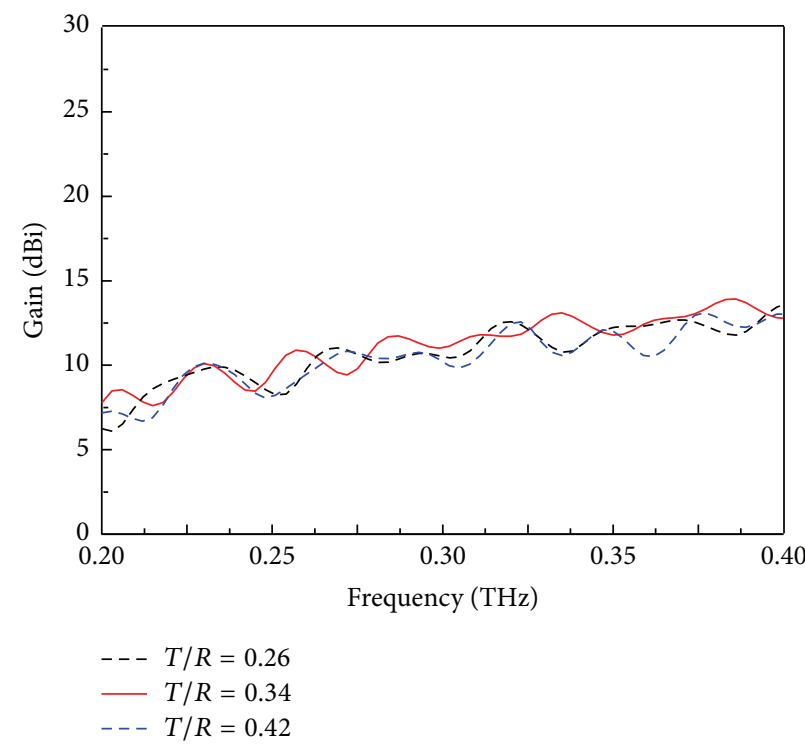

(a)

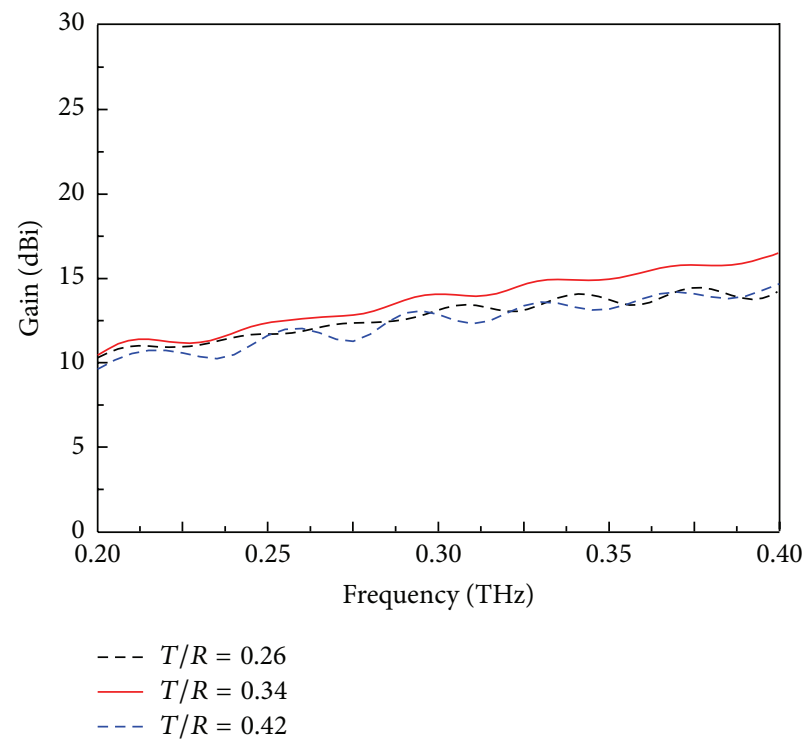

(b)

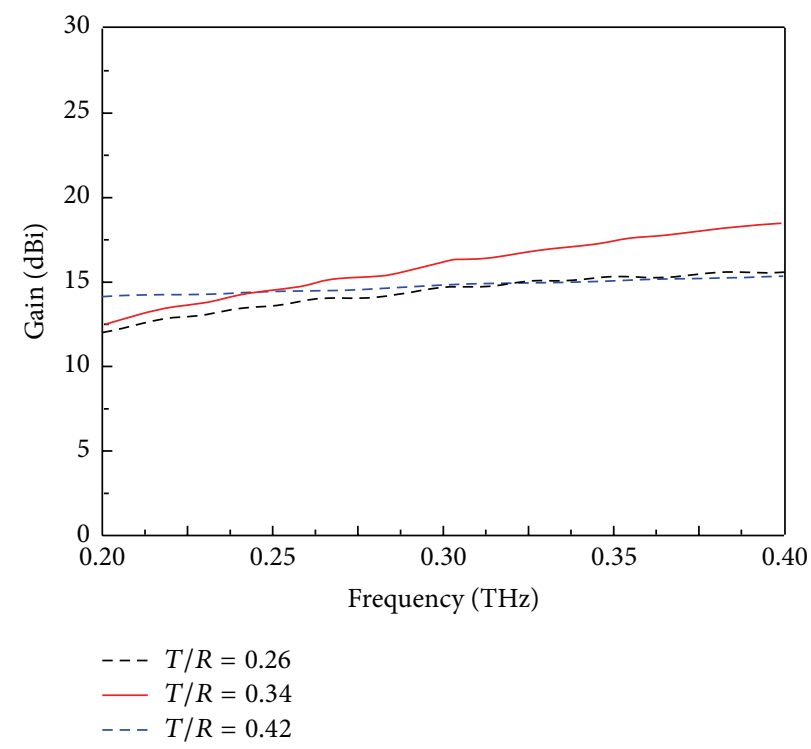

(c)

FIgURE 2: Comparison plots of antenna gain for different $T / R$ values with (a) $R=0.8 \lambda_{o}$, (b) $R=1.2 \lambda_{o}$, and (c) $R=1.6 \lambda_{o}$ compared to frequency.

of lens position with respect to the antenna [21, 22]. This confirms that the antenna gain is sensitive to the lens shape (determined by $T / R$ ), especially for the larger lenses at high frequencies $[12,23]$. Interestingly, the smaller lenses support a wider range of $T / R$ values in which good performance in terms of gain can be achieved and thus have an additional degree of freedom in device fabrication. In fact, a wide range of $T / R$ ratios can be obtained for a small lens as a result of a smaller change in $T$. This does not affect the optimized condition significantly. Nevertheless, the slit antenna showed the best gain curves for all lenses at $T / R=0.34$. In other words, it was found that when the $T / R$ ratio was 0.34 , the lens behaved as a superhemispherical lens. Thus, we maintained this optimized ratio of $T / R$ for each lens size in our study.
Figure 3(a) shows the antenna gain responses with different lens sizes with the fixed optimized ratio of $T / R=0.34$. The gain showed an increased level of dependency on the lens size, and it increased with the increasing lens radius. Improvements in the gain level were obtained by increasing the lens radius from $0.8 \lambda_{o}$ to $1.2 \lambda_{o}$ and from $1.2 \lambda_{o}$ to $1.6 \lambda_{o}$. With the smaller lens, the antenna did not perform well and showed low and unstable gain behavior. The lens with the radius of $0.8 \lambda_{o}$ produced a low and fluctuated gain; the fluctuation was very high at lower frequencies, and a reduction in fluctuation is seen at higher frequencies. For higher frequencies, the electrical length of the slit line is increased, which ensures the smooth decay of the current across the slit line, resulting in decreased fluctuations. The antenna gain 

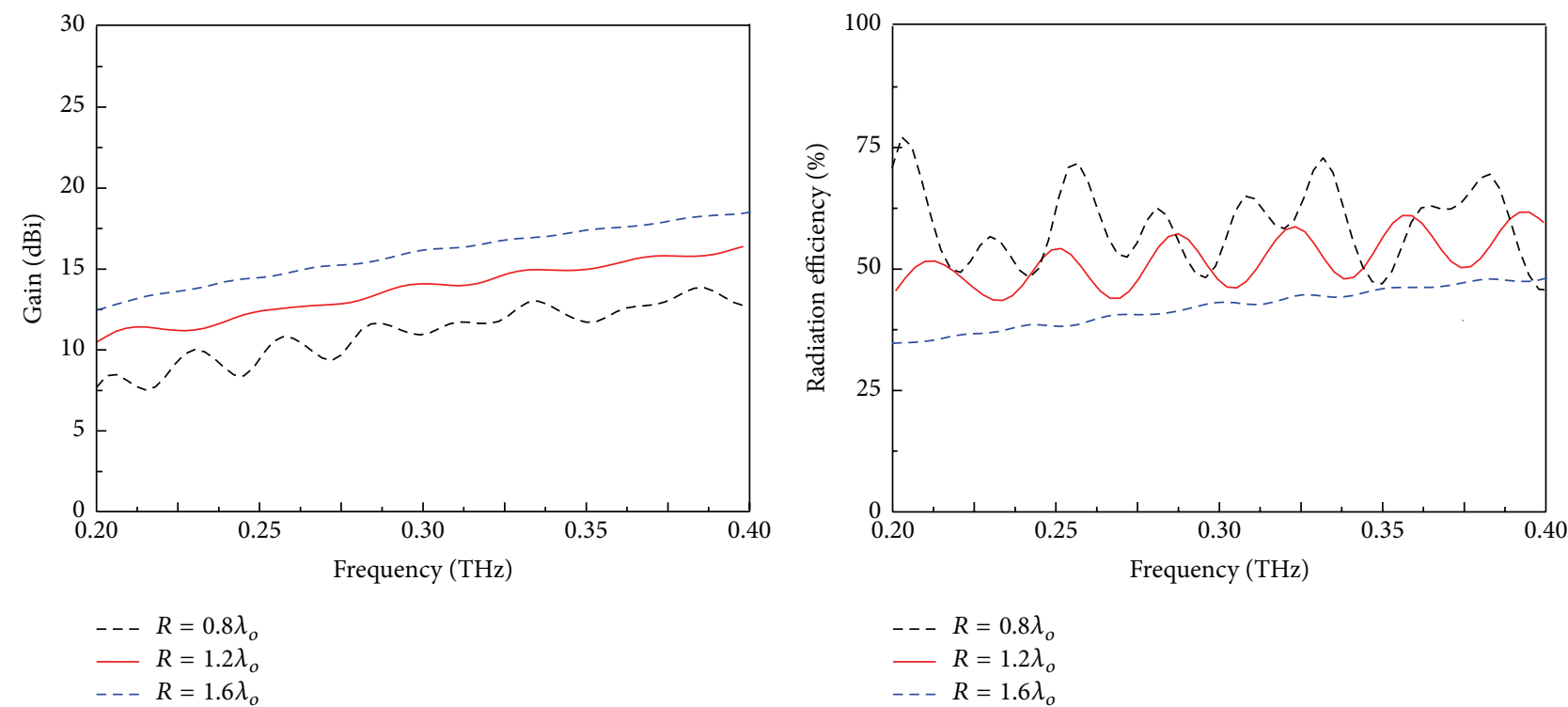

(a)

(b)

FIGURE 3: Comparison plots of (a) gain and (b) radiation efficiency for different lens radii with $T / R=0.34$ compared to frequency.

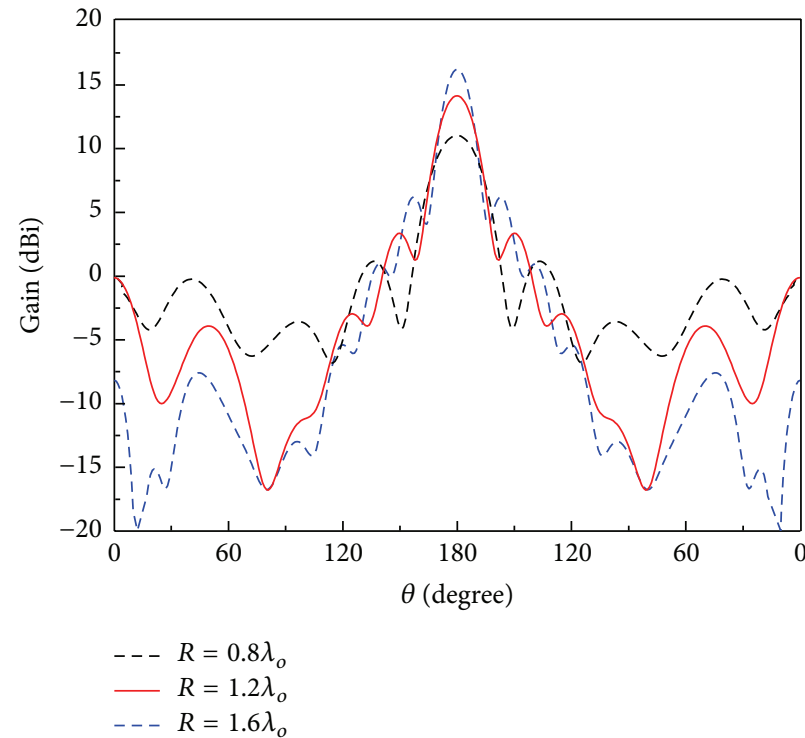

(a)

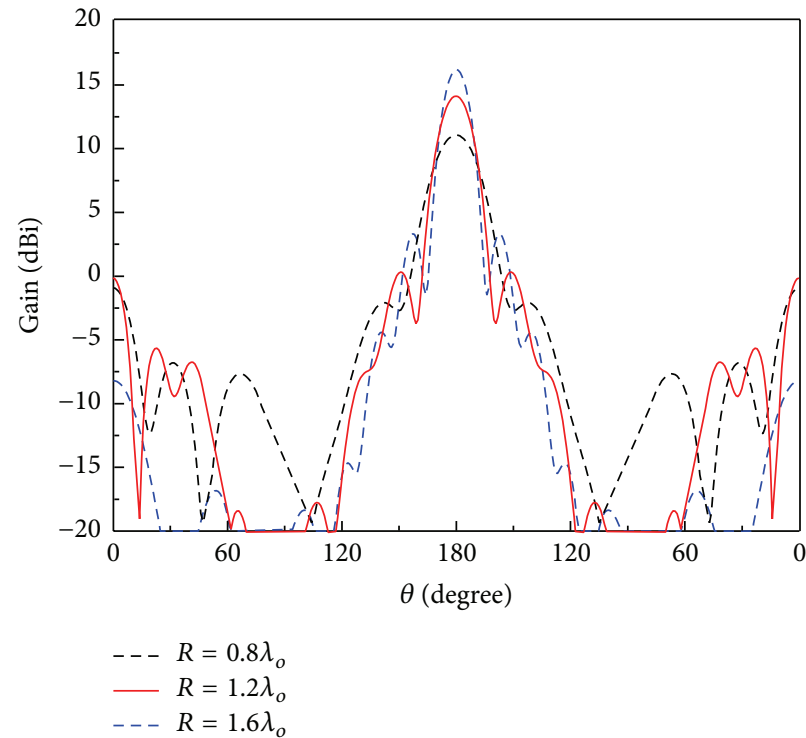

(b)

FIGURE 4: Radiation patterns of the antenna for different lens radii with fixed $T / R=0.34$ at $0.3 \mathrm{THz}$ : (a) $x z$-plane and (b) $y z$-plane.

was observed as $7.8 \mathrm{dBi}$ at $0.2 \mathrm{THz}$ and reached $12.7 \mathrm{dBi}$ at $0.4 \mathrm{THz}$. The gain of the antenna with the lens radius of $1.2 \lambda_{o}$ increased smoothly from $10.5 \mathrm{dBi}$ to $16.5 \mathrm{dBi}$, as the frequency increased from $0.2 \mathrm{THz}$ to $0.4 \mathrm{THz}$. Similarly, the lens with a radius of $1.6 \lambda_{o}$ produced the higher gain level of $12.5 \mathrm{dBi}$ at $0.2 \mathrm{THz}$, which gradually increased to $18.5 \mathrm{dBi}$ at $0.4 \mathrm{THz}$. The results confirm that, for each lens size, a low gain occurs at lower frequencies, and the antenna gain increases with frequency. This phenomenon is reasonable because for higher frequencies the effective size of the radiating element increases.
The radiation efficiency for each lens size was calculated and plotted as a function of the frequency, as shown in Figure $3(\mathrm{~b})$. The lens with the radius of $0.8 \lambda_{o}$ showed high radiation efficiency and had a high degree of fluctuations. Interestingly, both fluctuations and the radiation efficiency decreased with an increase in lens size. The lens with the radius of $1.2 \lambda_{o}$ gave a low fluctuated periodic radiation curve, while a lens with a $1.6 \lambda_{o}$ radius showed low but smooth and stable efficiency. The average efficiency decreased in decrements of approximately $10 \%$ for each increment of $0.4 \lambda_{o}$ in the lens radii. It was found that the average radiation 


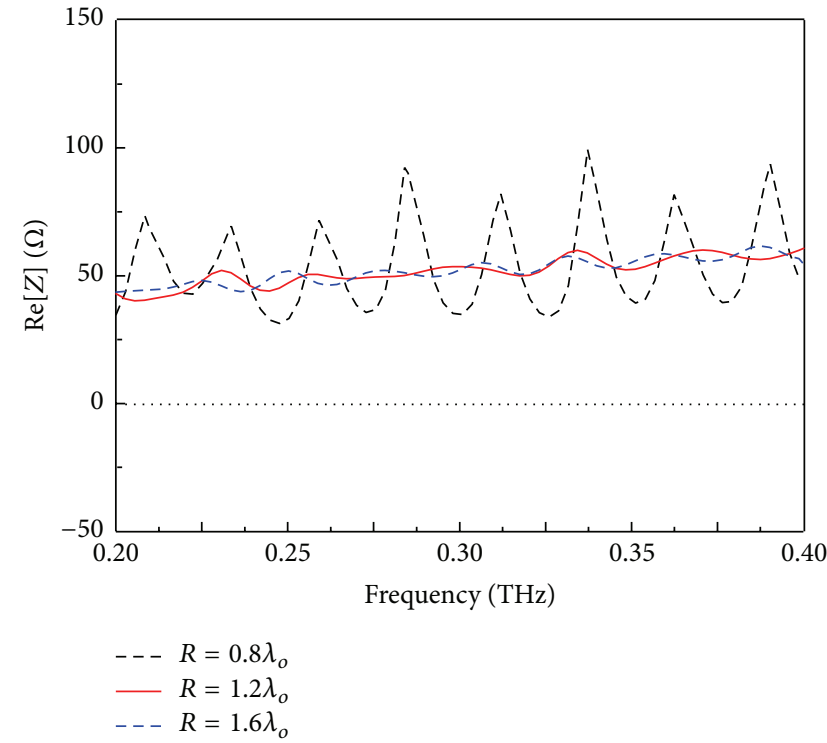

(a)

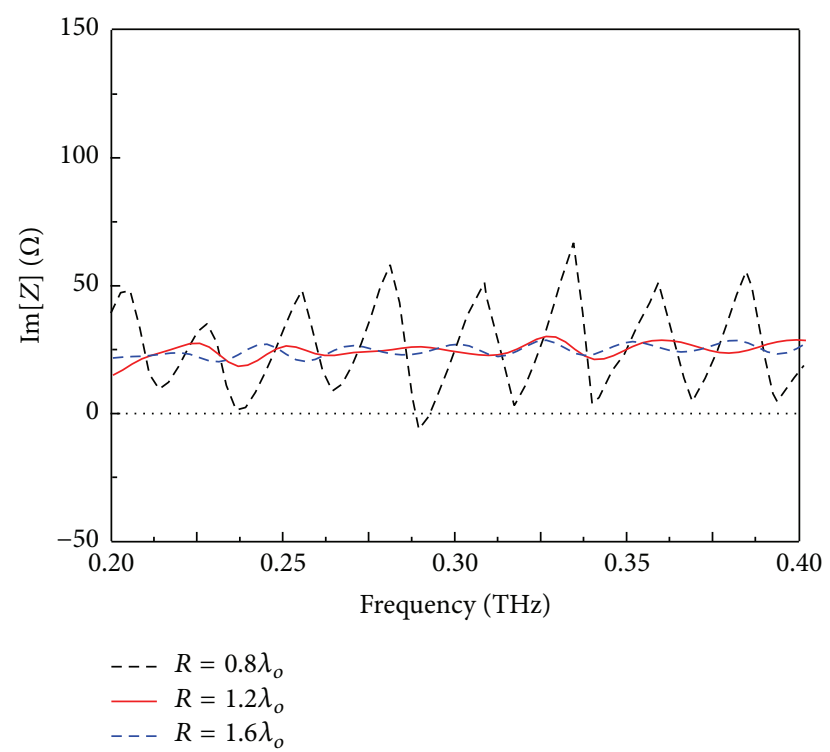

(b)

Figure 5: Comparison plots of input impedance versus frequency for different values of $R$ with $T / R=0.34$ : (a) real part and (b) imaginary part.

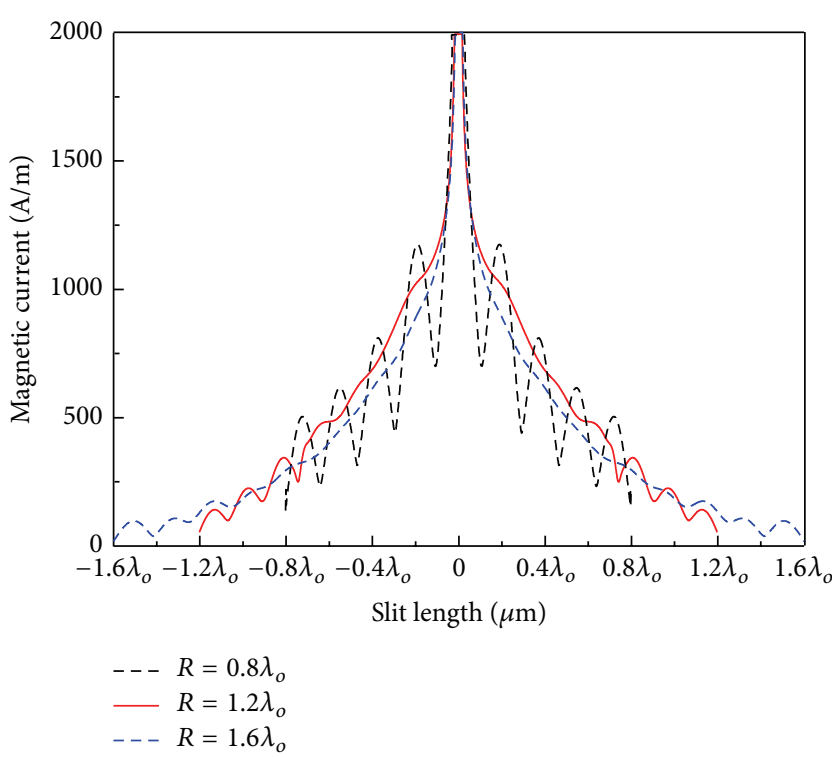

Figure 6: Comparison plots of current distribution for different values of $R$ with $T / R=0.34$ along the slit line at $0.3 \mathrm{THz}$.

efficiency is $61 \%$ for a lens radius of $0.8 \lambda_{o}$ and $52 \%$ and $41 \%$ for a lens radius of $1.2 \lambda_{o}$ and $1.6 \lambda_{o}$, respectively.

It is important to note that there is a trade-off between the gain and radiation efficiency with respect to the lens radius. A larger lens can produce high gain, but it has low radiation efficiency and a bulky size. Contrary to this, a lens with a small radius is capable of high radiation efficiency at the cost of reduced gain. However, for a slit dipole antenna, the lens with $R=1.2 \lambda_{o}$ produced a stable gain behavior with a good compromise in radiation efficiency.
The radiation patterns of the antenna on the different lens radii are plotted at the center frequency of $0.3 \mathrm{THz}$ in Figure 4. In general, the $y z$-plane ( $H$-plane) presented relatively clean profile patterns with few back and side lobes, while the $x z$-plane (E-plane) showed more back and side lobes. The number of back and side lobes in both principal planes increased with an increase in lens radius. This is because of the leaky-wave nature of the slit dipole antenna; the slit line length increased for the lens of larger radii, which created a longer traveling wave path along the slit line. Furthermore, we saw a narrowing of the main beam and an increase in a gain level with the increasing lens radius. This is because of the increasing aperture size of the lens and thus the enhancement of the beam collimation. The side lobe levels in the $x z$-plane for all lenses remained the same at about $-10.2 \mathrm{~dB}$, whereas, in the $y z$-plane, it was $-10.2 \mathrm{~dB},-13.8 \mathrm{~dB}$, and $-12.8 \mathrm{~dB}$ for lens radii of $0.8 \lambda_{o}, 1.2 \lambda_{o}$, and $1.6 \lambda_{o}$, respectively.

The back radiations of lens-coupled antennas are inevitable. The back radiations were higher for smaller lenses and lower for the larger lens. Because the ratio of power between dielectric and air for the slit structure is $\varepsilon_{r}^{3 / 2}$, where $\varepsilon_{r}$ is the relative dielectric constant of the lens, the back lobes may be considerably alleviated using lens material with much higher $\varepsilon_{r}$ values or by further increasing the lens size.

The input impedance of the slit dipole antenna for different values of $R$ is illustrated in Figure 5. The high degree of fluctuation in the input impedance decreased with the increasing lens radius. The high fluctuation in the lens of $R$ $=0.8 \lambda_{0}$ decreased to a stable and constant impedance at lens sizes $R=1.2 \lambda_{o}$ and $R=1.6 \lambda_{o}$. The larger lenses enabled the design of a longer slit and thus reduced the current reflect back at slit termination. The real parts of the input impedance for $R=1.2 \lambda_{o}$ and $R=1.6 \lambda_{o}$ were around $50 \Omega$, while the imaginary parts of the input impedance were around $25 \Omega$ in 


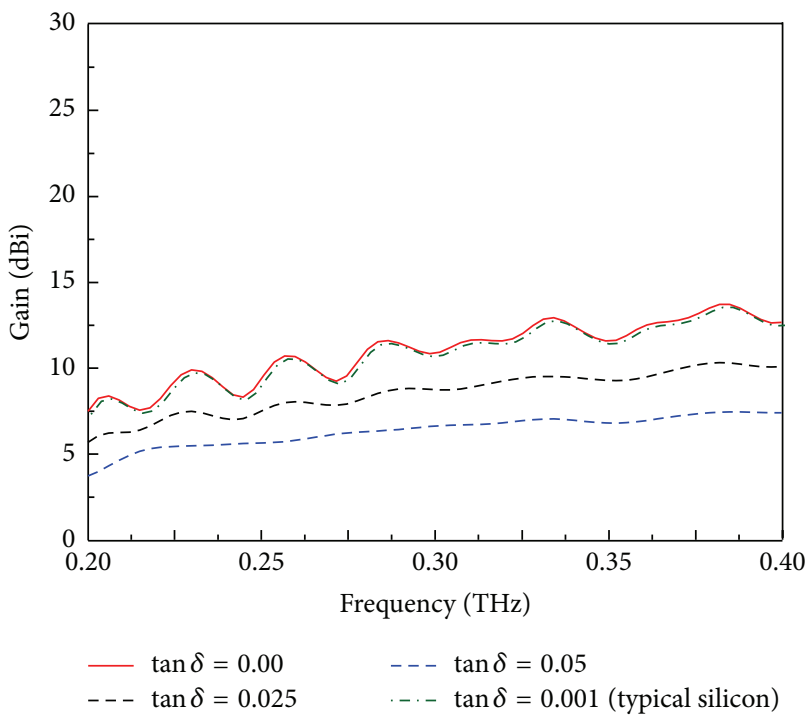

(a)

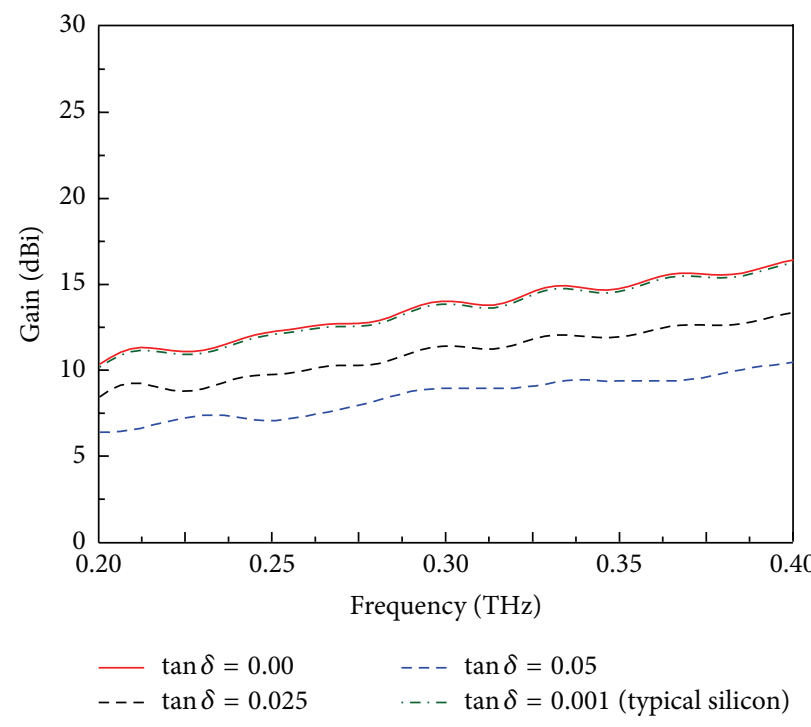

(b)

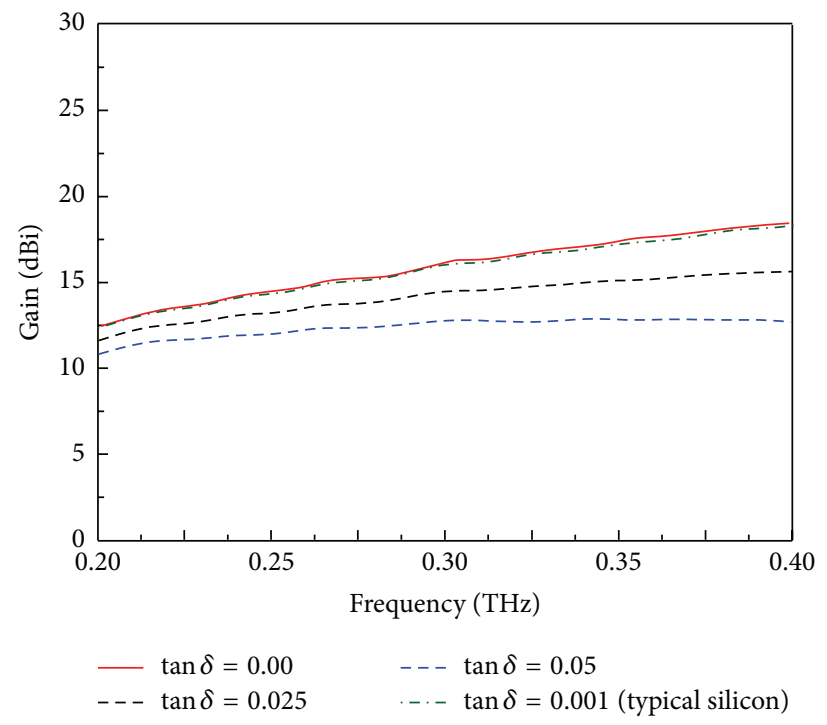

(c)

FIGURE 7: Comparison plots of antenna gain for silicon lenses with different values of loss tangent $\left(\tan \delta\right.$ ) for (a) $R=0.8 \lambda_{o}$, (b) $R=1.2 \lambda_{o}$, and (c) $R=1.6 \lambda_{o}$.

the entire frequency range. We calculated a magnetic current along the slit line arms in Figure 6 . As the length of the slit line increased with the increasing radius of the lens, the current which reflected back at the ends of the slit line decreased. The lens with the radius of $0.8 \lambda_{o}$ showed significant reflection at the slit termination. However, most of the current traveling through the slit line was attenuated rapidly and reached nearly zero at the slit termination for lens radii of $1.2 \lambda_{o}$ and $1.6 \lambda_{o}$. This verifies the leaky-wave nature of the slit dipole antenna, particularly with larger lenses.

The loss levels incurred by the dielectric lenses might have a significant impact on the antenna's performance. The effect of various values of loss tangents $(\tan \delta$ ) of silicon on the antenna's gain performance has been studied, as shown in Figure 7. At first, the loss tangent was assumed to be zero and then increased to 0.025 and 0.05 . The gain level decreased in the entire frequency range of interest for all lenses as the loss tangent value increased. Moreover, the gain fluctuations decreased as the loss tangent value increased; this was more significant for smaller lenses. However, the typical loss tangent value of high-resistivity silicon lens is $\tan \delta=0.001$ at around $0.3 \mathrm{THz}$ [24]. The results with this typical loss tangent value of silicon were almost the same as the results with $\tan \delta=0.0$ for all lenses, as shown in Figure 7.

It was important to understand how antenna's performance is affected by other lossy low dielectric constant materials. The antenna's performance with respect to gain is analyzed for quartz $\left(\varepsilon_{r}=3.8\right.$ and $\left.\tan \delta=0.0005\right)$ lenses for different $T / R$ values of $R=0.8 \lambda_{o}, R=1.2 \lambda_{o}$, and $R=1.6 \lambda_{o}$ 


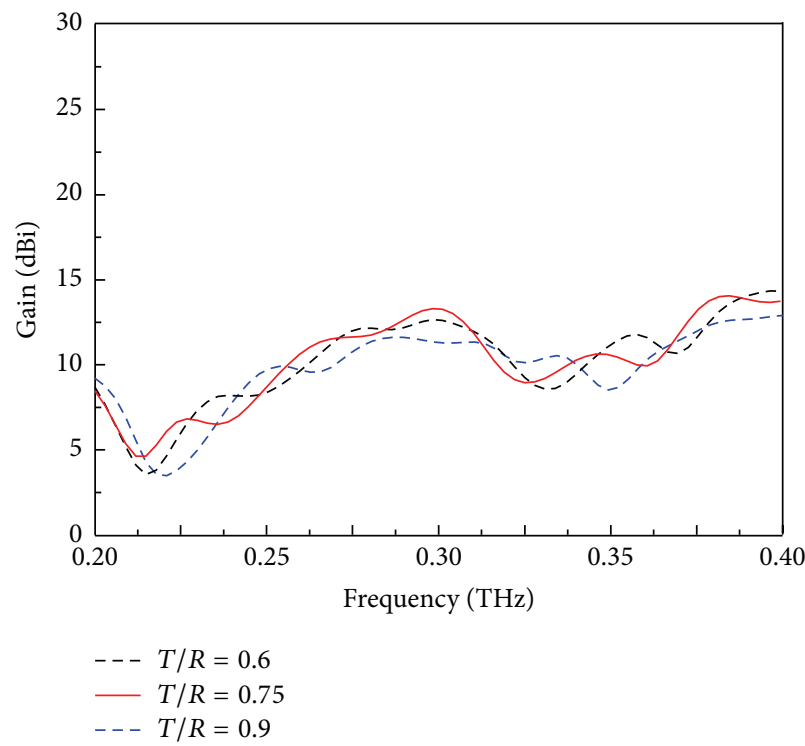

(a)

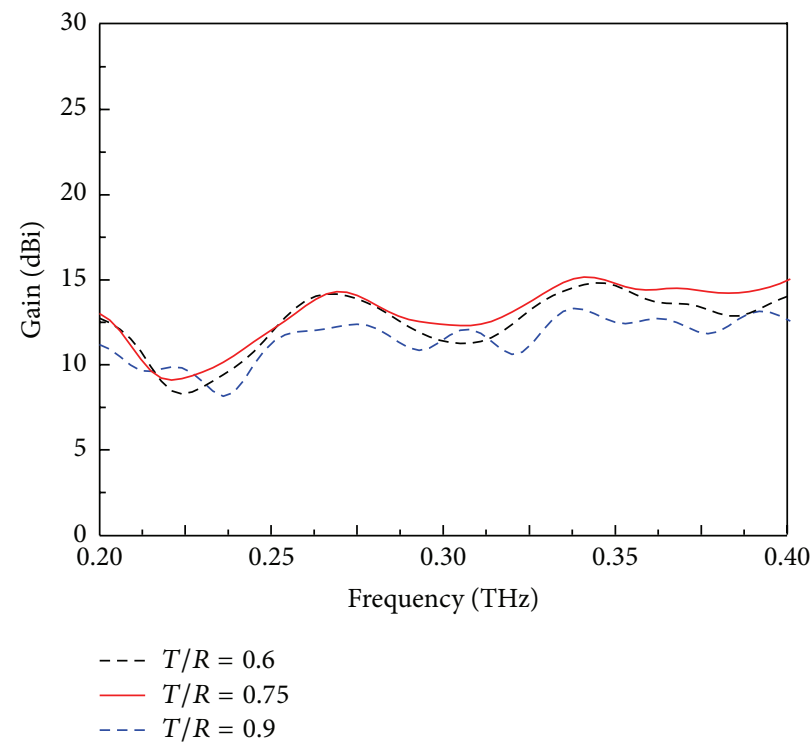

(b)

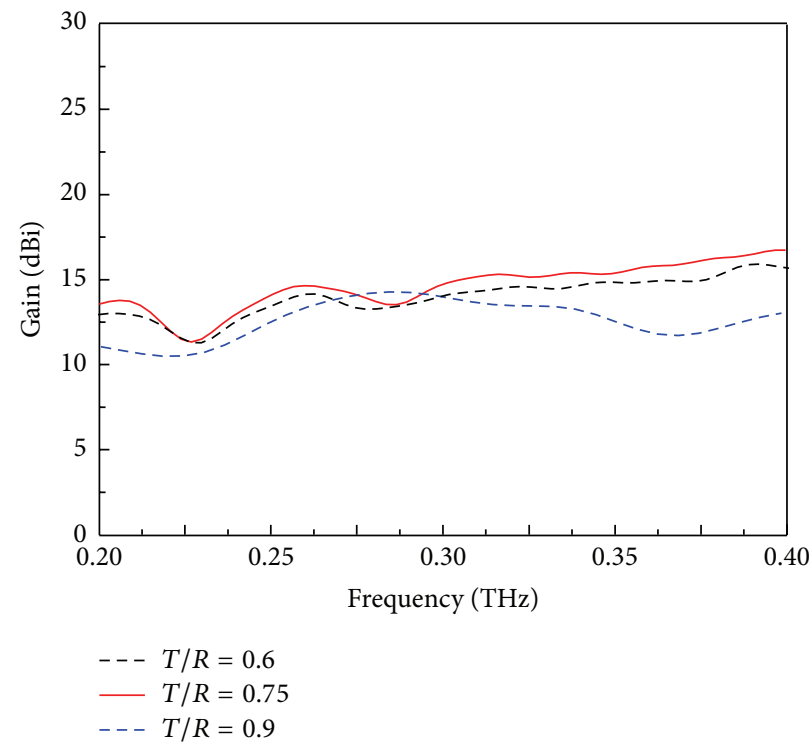

(c)

Figure 8: Comparison plots of antenna gain for quartz lenses with different $T / R$ values of (a) $R=0.8 \lambda_{o}$, (b) $R=1.2 \lambda_{o}$, and (c) $R=1.6 \lambda_{o}$.

in Figure 8. The smaller lenses $\left(R=0.8 \lambda_{o}\right.$ and $\left.R=1.2 \lambda_{o}\right)$ did not work properly, exhibiting low and fluctuating gain curves. The lens with $R=1.6 \lambda_{o}$ showed better performance but significant fluctuation at lower frequencies. It is important to note that the optimized $T / R$ ratio was 0.75 for quartz, which was only 0.34 in the case of silicon lens. Besides providing a compact lens size, an extended hemispherical lens with a high dielectric constant material (e.g., silicon) performs better than the low dielectric constant lens due to the fact that high dielectric constant material yields a more exact geometrical approximation of an ellipse [25]. Thus, silicon is the best candidate for the design of a minimum lens size supporting the leaky-wave nature of the slit dipole antenna.

\section{Conclusions}

The influence of lens shape and size on the characteristics of the slit dipole antenna was studied over a broad frequency range around $0.3 \mathrm{THz}$ to find the smallest lens that supports the leaky-wave behavior of the slit dipole antenna. The results showed that the lens size and shape play a key role in determining the best possible gain and radiation spectral bandwidth of the antenna. The gain response was found to be sensitive and changed with the change in lens size; it increased with an increasing lens size over the entire frequency range of interest. The results showed that the lens with the radius of $1.2 \lambda_{o}(1.2 \mathrm{~mm})$ at $0.3 \mathrm{THz}$ is the smallest lens in which a slit dipole antenna on the extended hemispherical silicon 
lens performs as a leaky-wave antenna. Further decreasing the lens size leads to more fluctuations in gain and input impedance, and the smooth gain and input impedance curves vanish.

\section{Competing Interests}

The authors declare that they have no competing interests.

\section{Acknowledgments}

This work was supported by the ICT R\&D Program of MSIP/IITP [14-911-01-001].

\section{References}

[1] I. F. Akyildiz, J. M. Jornet, and C. Han, "Terahertz band: next frontier for wireless communications," Physical Communication, vol. 12, pp. 16-32, 2014.

[2] H. J. Song, K. Ajito, Y. Muramoto, A. Wakatsuki, T. Nagatsuma, and N. Kukutsu, "Gbit/s data transmission in $300 \mathrm{GHz}$ band for future terahertz communications," Electronics Letters, vol. 48, no. 15, pp. 953-954, 2012.

[3] P. H. Siegel, "Terahertz technology in biology and medicine," IEEE Transactions on Microwave Theory and Techniques, vol. 52, no. 10, pp. 2438-2447, 2004.

[4] D. B. Rutledge and M. S. Muha, "Imaging antenna arrays," IEEE Transactions on Antennas and Propagation, vol. 30, no. 4, pp. 535-540, 1982.

[5] D. M. Mittleman, M. Gupta, R. Neelamani, R. G. Baraniuk, J. V. Rudd, and M. Koch, "Recent advances in terahertz imaging," Applied Physics B, vol. 68, no. 6, pp. 1085-1094, 1999.

[6] T. K. Nguyen, H. Han, and I. Park, "Full-wavelength dipole antenna on a hybrid GaAs membrane and Si lens for a terahertz photomixer," Journal of Infrared, Millimeter, and Terahertz Waves, vol. 33, no. 3, pp. 333-347, 2012.

[7] J. Klier, G. Torosyan, N. S. Schreiner et al., "Influence of substrate material on radiation characteristics of $\mathrm{THz}$ photoconductive emitters," International Journal of Antennas and Propagation, vol. 2015, Article ID 540175, 7 pages, 2015.

[8] H. Liu, J. Yu, P. Huggard, and B. Alderman, "A multichannel $\mathrm{THz}$ detector using integrated bow-tie antennas," International Journal of Antennas and Propagation, vol. 2013, Article ID 417108, 8 pages, 2013.

[9] Z. Jiang, S. Rahman, J. L. Hesler, P. Fay, and L. Liu, "Design and characterization of tunable lens-coupled annular-slot antennas for all-electronic reconfigurable $\mathrm{THz}$ detectors and focal-plane arrays," IEEE Xplore: IET Microwaves, Antennas \& Propagation, vol. 8, no. 11, pp. 842-848, 2014.

[10] O. M. Haraz, A. R. Sebak, and S. Alshebeili, "Study the effect of using low-cost dielectric lenses with printed log-periodic dipole antennas for millimeter-wave applications," International Journal of Antennas and Propagation, vol. 2015, Article ID 209430, 7 pages, 2015.

[11] S. M. Rahman, Z. Jiang, H. Xing, P. Fay, and L. Liu, "Lenscoupled folded-dipole antennas for terahertz detection and imaging," IET Microwaves, Antennas and Propagation, vol. 9, no. 11, pp. 1213-1220, 2015.

[12] A. A. Oliner and D. R. Jackson, "Leaky-wave antennas," in Antenna Engineering Handbook, chapter 11, McGraw-Hill, New York, NY, USA, 4th edition, 2007.
[13] T. K. Nguyen, F. Rotermund, and I. Park, "A traveling-wave stripline dipole antenna on a substrate lens at terahertz frequency," Current Applied Physics, vol. 14, no. 8, pp. 998-1004, 2014.

[14] A. Neto, "UWB, non dispersive radiation from the planarly fed leaky lens antenna-part I: theory and design," IEEE Transactions on Antennas \& Propagation, vol. 58, no. 7, pp. 2238-2247, 2010.

[15] N. Llombart, G. Chattopadhyay, A. Skalare, and I. Mehdi, "Novel terahertz antenna based on a silicon lens fed by a leaky wave enhanced waveguide," IEEE Transactions on Antennas and Propagation, vol. 59, no. 6, pp. 2160-2168, 2011.

[16] O. Yurduseven, D. Cavallo, A. Neto, G. Carluccio, and M. Albani, "Parametric analysis of extended hemispherical dielectric lenses fed by a broadband connected array of leaky-wave slots," IET Microwaves, Antennas and Propagation, vol. 9, no. 7, pp. 611-617, 2015.

[17] CST Microwave Studio, CST GmbH, 2012, https://www.cst.com/.

[18] T. K. Nguyen, B. Q. Ta, and I. Park, "Design of a planar, high-gain, substrate-integrated Fabry-Perot cavity antenna at terahertz frequency," Current Applied Physics, vol. 15, no. 9, pp. 1047-1053, 2015.

[19] A. Neto, S. Bruni, G. Gerini, and M. Sabbadini, "The leaky lens: a broad-band fixed-beam leaky-wave antenna," IEEE Transactions on Antennas and Propagation, vol. 53, no. 10, pp. 3240-3246, 2005.

[20] N. Llombart and A. Neto, "THz time-domain sensing: the antenna dispersion problem and a possible solution," IEEE Transactions on Terahertz Science and Technology, vol. 2, no. 4, pp. 416-423, 2012.

[21] H. Kobayashi and Y. Yasuoka, "Receiving properties of extended hemispherical lens coupled slot antennas for $94-\mathrm{GHz}$ millimeter wave radiation," Electronics and Communications in Japan, Part I, vol. 84, no. 6, pp. 32-40, 2001.

[22] P. Nenzi, V. Varlamava, F. S. Marzano, F. Palma, and M. Balucani, "Dielectric lens optimization for conical helix THz antennas," in Proceedings of the 64th Electronic Components and Technology Conference (ECTC '14), pp. 2137-2143, IEEE, Orlando, Fla, USA, May 2014.

[23] T. K. Nguyen, T. A. Ho, H. Han, and I. Park, "Numerical study of self-complementary antenna characteristics on substrate lenses at terahertz frequency," Journal of Infrared, Millimeter, and Terahertz Waves, vol. 33, no. 11, pp. 1123-1137, 2012.

[24] P. H. Bolivar, M. Brucherseifer, J. G. Rivas et al., "Measurement of the dielectric constant and loss tangent of high dielectricconstant materials at terahertz frequencies," IEEE Transactions on Microwave Theory and Techniques, vol. 51, no. 4, pp. 10621066, 2003.

[25] D. F. Filipovic, S. S. Gearhart, and G. M. Rebeiz, "Doubleslot antennas on extended hemispherical and elliptical silicon dielectric lenses," IEEE Transactions on Microwave Theory and Techniques, vol. 41, no. 10, pp. 1738-1749, 1993. 


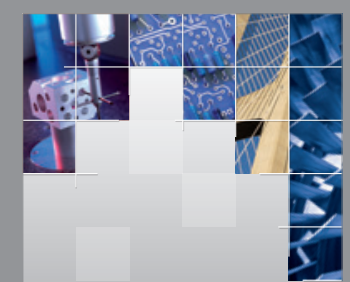

\section{Enfincering}
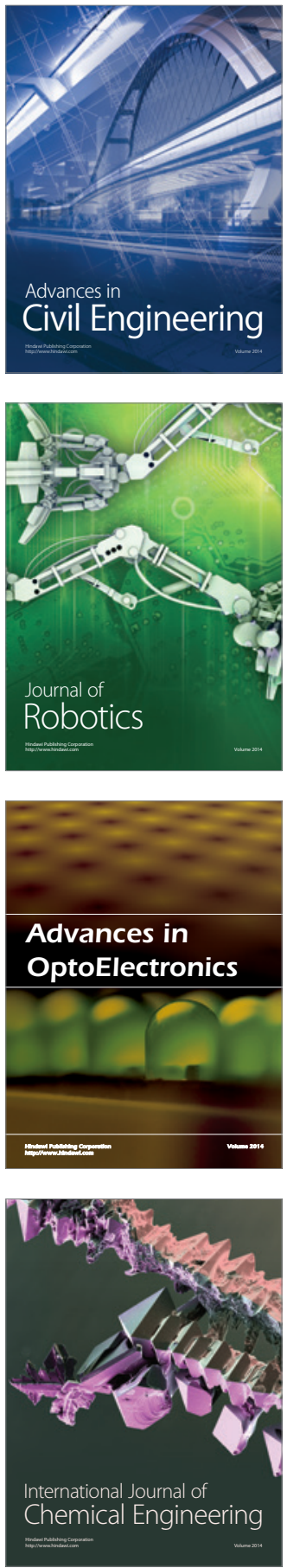

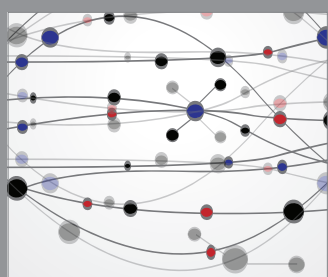

The Scientific World Journal

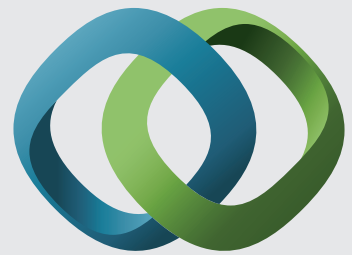

\section{Hindawi}

Submit your manuscripts at

http://www.hindawi.com
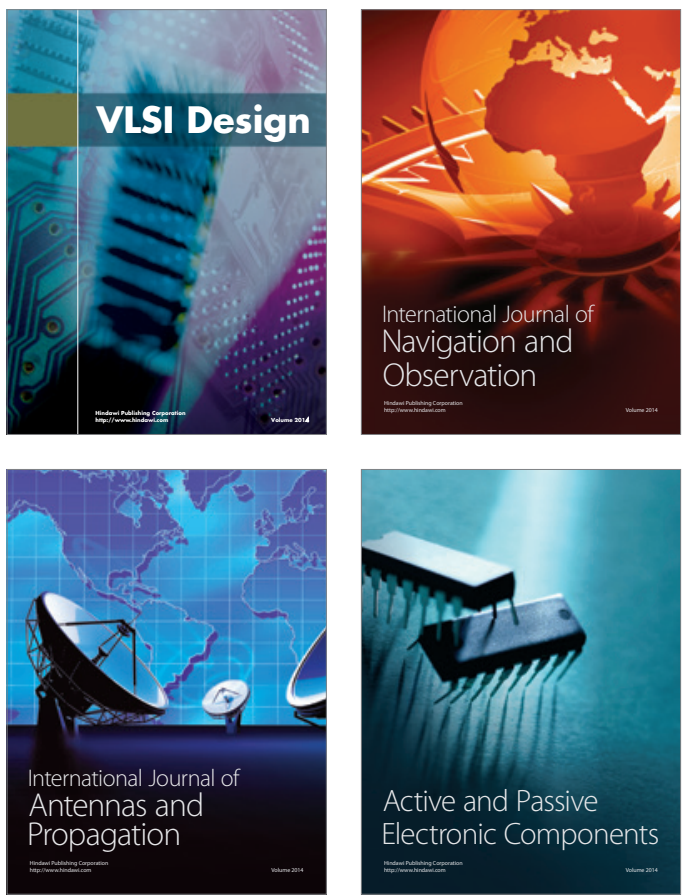
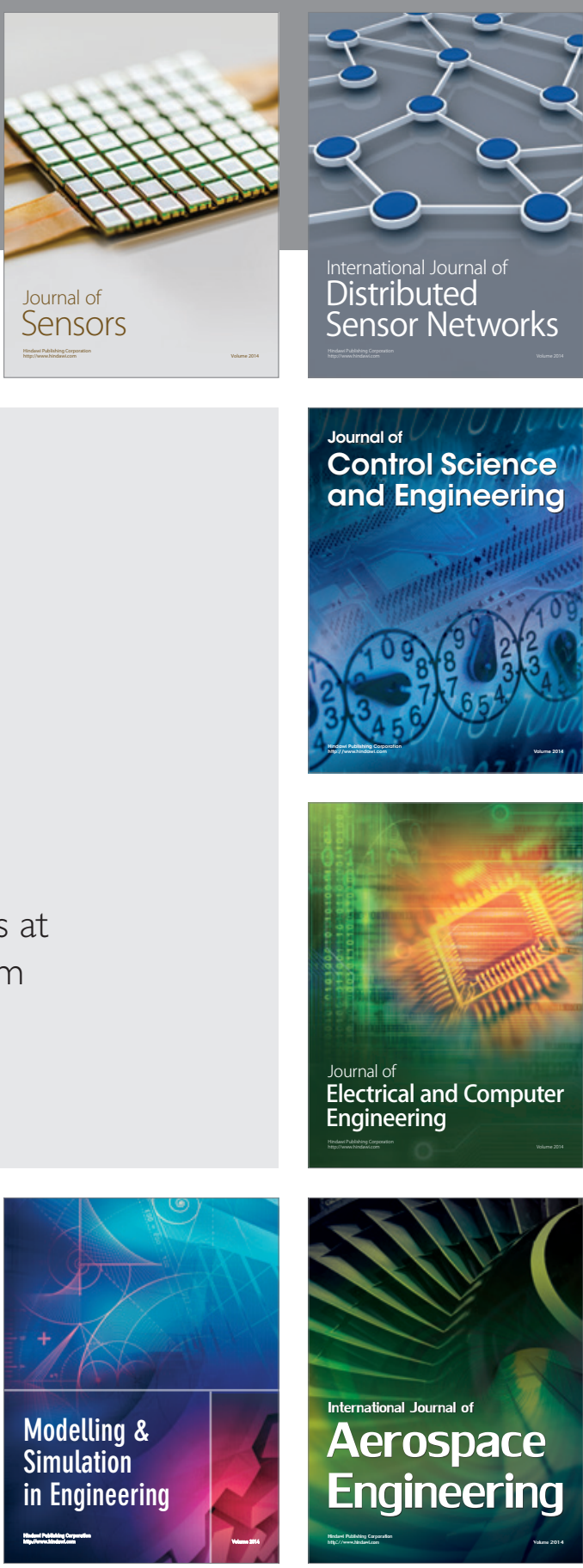

International Journal of

Distributed

Sensor Networks

Journal of

Control Science

and Engineering
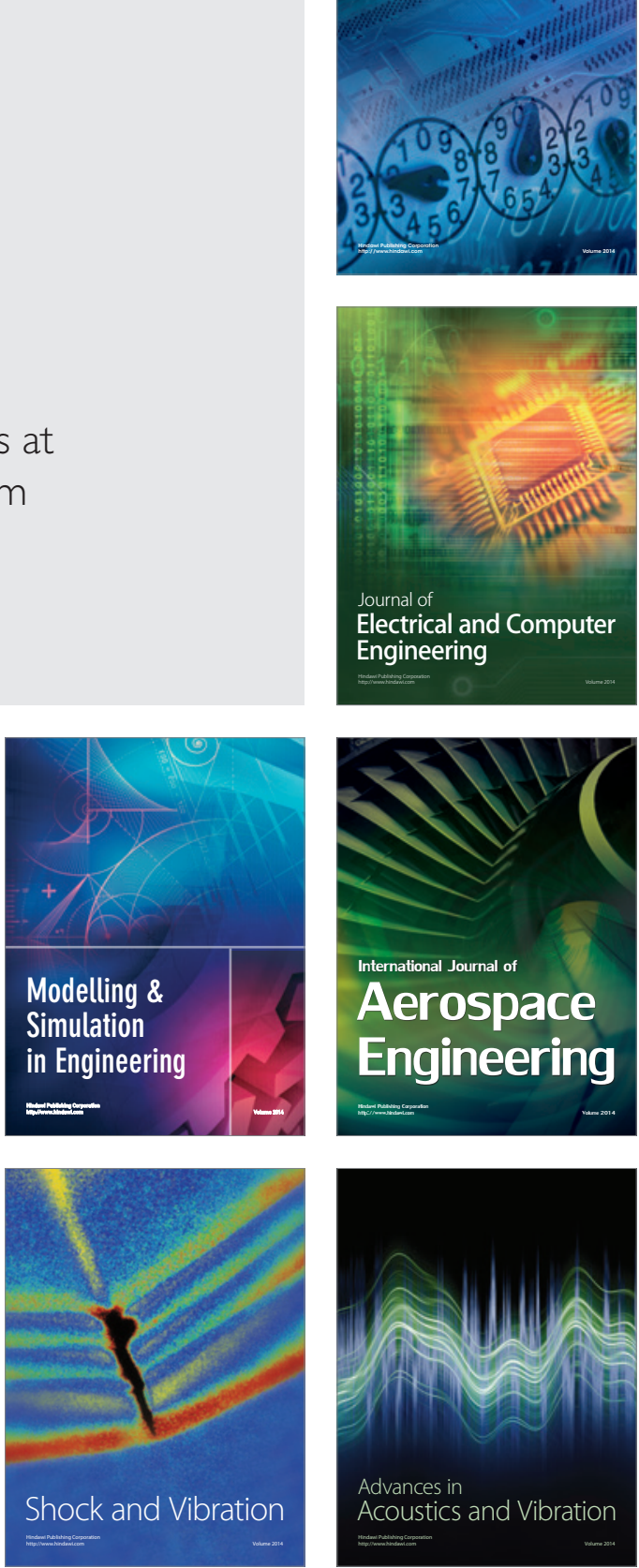